\title{
Design and Implementation of Fiber-embedded Plasmonic Structures in Microwires
}

\author{
A. Petropoulou ${ }^{1,4}$, G. Antonopoulos ${ }^{1}$, P. Bastock ${ }^{2}$, G. Kakarantzas ${ }^{1}$, C. Craig ${ }^{2}$, \\ D. Drikakis ${ }^{3}$, D. W. Hewak ${ }^{2}$, M. N. Zervas ${ }^{2}$, and C. Riziotis ${ }^{1}$
}

\author{
${ }^{1}$ Theoretical and Physical Chemistry Institute \\ National Hellenic Research Foundation, Athens 116 35, Greece \\ ${ }^{2}$ Optoelectronics Research Centre, University of Southampton, Southampton SO17 1BJ, United Kingdom \\ ${ }^{3}$ School of Sciences and Engineering \& Medical School, University of Nicosia, Nicosia CY 2417, Cyprus \\ ${ }^{4}$ Department of Informatics and Telecommunications, University of Peloponnese, Tripolis 22100, Greece
}

\begin{abstract}
Plasmonic structures can dramatically enhance photonic devices functionality [1] by providing controllable field confinement and light nanofocussing which are crucial for imaging, diagnostic, and sensing applications. Pure metallic tips or metal coated optical fibers have been demonstrated as fiber-compatible efficient plasmonic devices [2] but with limited applicability in real applications due to fragility and limited environmental robustness.

The proposed platform based on hybrid microwires composed of metal core and silicate glass cladding offers the required robustness and flexibility for engineering and developing plasmonic devices in all-fiber form [3]. The presence of the dielectric cladding offers continuous re-excitation of the plasmon modes due to repeated total internal reflection at the glass/air interface, which can dramatically reduce the high losses induced by the metal core and allow long propagation distances. This enables direct light coupling from the distal end of fiber instead of side excitation of the tip, allowing their integration in optical fiber or and planar integrated circuitry for hybrid architectures. By employing the heating and stretching thermal processing method for diameter tapering of microwires with gold core, high-quality all-fiber plasmonic tips with high field intensity at the tip apex have been fabricated. Furthermore, embedded metal microspheres, as seen in the figure, were controllably formed targeting to the development of in-fiber plasmonic resonators.
\end{abstract}

Extensive theoretical and experimental investigations were necessary for the identification of appropriate tapering conditions and adiabatic metal tips development with well-defined geometrical characteristics. In this context, analytical studies and microfluidic simulations by Finite Element Method - FEM were performed for the understanding of the appropriate thermal processing conditions of microwires and their behaviour towards their diameter tapering without discontinuities and metal core breakage. Fabricated plasmonic tips performance was successfully related to simulation results by FEM, predicting high field enhancement factors up to $10^{5}$. Furthermore, theoretical investigations of instabilities-driven formation of gold microspheres embedded in the glass cladding by heating the hybrid microfibers was also performed suggesting ways to control the spherical formed features.
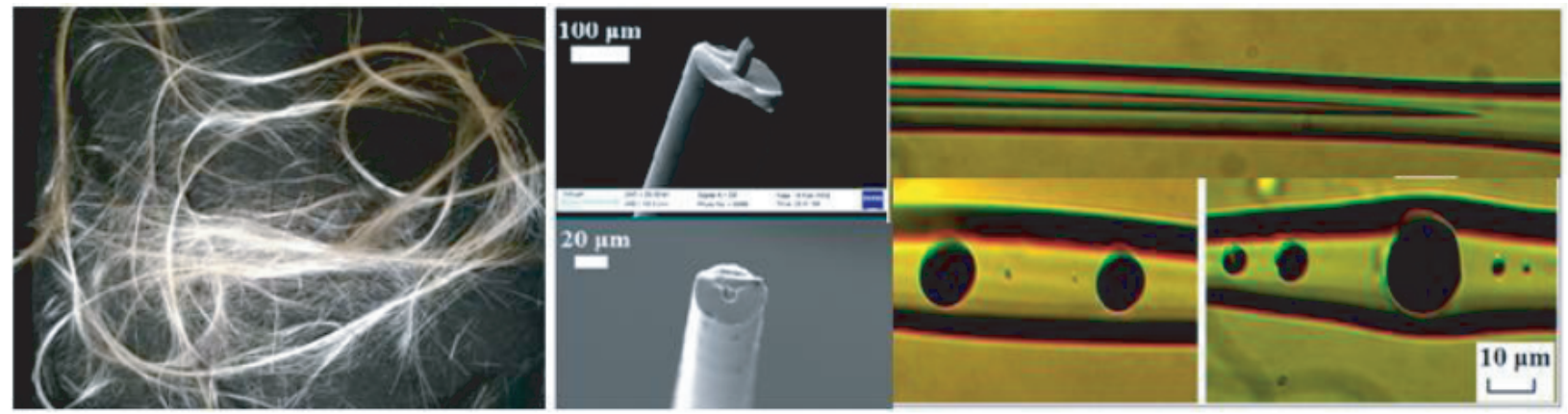

Figure 1: Pristine gold microwires. Microwires' SEM characterization. Tapered tip and formed microspheres [3]. 


\section{REFERENCES}

1. Riziotis, C., V. Pruneri, P. G. R. Smith, and A. Vasilakos, "Fiber and integrated waveguidebased optical sensors," Journal of Sensors, Article ID 171748, 2009.

2. Petropoulou, A., M. N. Zervas, and C. Riziotis, "Design optimization of gold-coated fiber tips with embedded plasmonic slot nano-resonators," Journal of Optics, Vol. 19, 055002, 2017.

3. Petropoulou, A., G. Antonopoulos, P. Bastock, G. Kakarantzas, C. Craig, D. W. Hewak, M. N. Zervas, and C. Riziotis, "All-fiber plasmonic platform based on hybrid composite metal/glass microwires," J. Phys. Chem. C, Vol. 22, No. 45, 26169-26176, 2018. 